
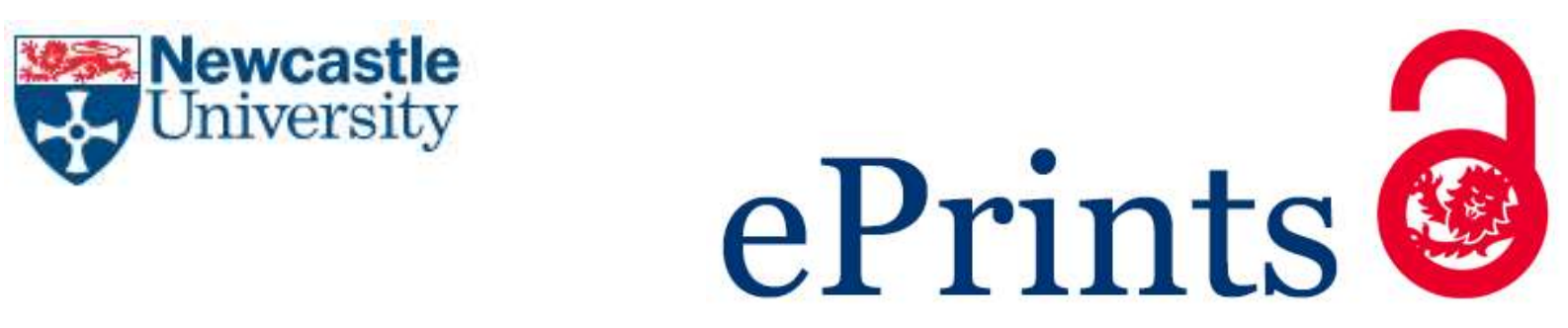

Yang W, Lang Z, Tian W. Condition Monitoring and Damage Location of Wind Turbine Blades by Frequency Response Transmissibility Analysis. IEEE Transactions on Industrial Electronics 2015, 62(10), 6558-6564.

\title{
Copyright:
}

(C) 2016 IEEE. Personal use of this material is permitted. Permission from IEEE must be obtained for all other uses, in any current or future media, including reprinting/republishing this material for advertising or promotional purposes, creating new collective works, for resale or redistribution to servers or lists, or reuse of any copyrighted component of this work in other works.

DOI link to article:

http://dx.doi.org/10.1109/TIE.2015.2418738

Date deposited:

$25 / 08 / 2016$ 


\section{Condition Monitoring and Damage Location of Wind Turbine Blades by Frequency Response Transmissibility Analysis}

\begin{abstract}
Incipient defects occurring in long wind turbine (WT) blades are difficult to detect using the existing condition monitoring (CM) techniques. To tackle this issue, a new WT blade CM method is studied in this paper with the aid of the concept of the transmissibility of Frequency Response Functions (FRFs). Different from the existing CM techniques that judge the health condition of a blade by interpreting individual CM signals, the proposed method jointly utilizes the CM signals measured by a number of neighboring sensors. This offers the proposed technique a unique capability of both damage detection and location. The proposed technique has been experimentally verified by using the real $\mathrm{CM}$ data collected during the fatigue and static tests of a full scale WT blade. Experiment has shown that the new technique is effective not only in damage detection but in damage location when either Fiber Bragg Grating (FBG) strain gauges or accelerometers are used for data acquisition.
\end{abstract}

Index Terms - wind turbine, blade, transmissibility analysis, condition monitoring.

\section{INTRODUCTION}

Wind turbine (WT) blades capture energy from wind and convert it to mechanical energy for electricity power generation. They are therefore one of the most critical components in a WT system. However, WT blades are exposed to direct harsh environment, suffering constantly varying loads, experiencing temperature and humidity changes, erosion, and corrosion in operation. They are also the most vulnerable component in a WT. The long-term practice has shown that blade failures account for about $10 \%$ of all WT failures that have been reported [1]. These blade failures lead to over $15 \%$ of total downtime [2], which means a significant revenue loss to the operator. Moreover, a catastrophic failure of blade could result in the loss of the whole turbine or even casualty and damage to neighboring facilities in the wind farm. Thus, blade failures have a profound impact on the cost of energy from wind [4]. For this reason, to detect the failure of blade as early as possible and take measures to protect those being defective is of great significance in increasing the availability and therefore the economic return of WTs.

As mentioned in [5], at present onsite visual inspections at regular intervals are being popularly used for blade condition monitoring (CM). Such an approach can provide 'snapshots' of the actual health condition of the blades. It is however unlikely to carry out in unfavourable sea and weather conditions. Moreover, onsite inspection of blade requires specialized equipment (e.g., cranes and working platforms) and expert personnel, which will consume lots of time and money. In offshore circumstance, onsite inspection will become more problematic and expensive due to the limited access to rough sea and additional costs on vessels and other tools specially required in the work on sea. For these reasons, online CM is strongly recommended.

In the last decades, efforts have been made to develop various online CM techniques dedicatedly for WT blades [6][7][8][9][10][11], some of which are already commercialized (e.g., Moog Insensys' rotor monitoring system designed based on optical fibre sensors [12]) while others are still in research (e.g. vibration analysis [5], electrical resistance [6], acoustic emission [13], Ultrasonic wave [14], etc.). Recently, a few cost-effective blade CM techniques were reported, for example [15] used the generator stator current signal to monitor the health conditions of blades in a permanent magnet direct-drive WT; [9] and [16] detected blade failure by interpreting the data collected by WT SCADA system. All these efforts have advanced the blade CM technology to certain extent. However, they have not provided a fully successful tool for predicting the actual health condition of WT blades particularly those with large sizes. The reasons can be various, but the major ones can be summarized as follows:

- The incipient defect cannot lead to significant change in the structural integrity of the blade. As a consequence, the bending moment measured at the root section of a blade [12] or the dynamic response indicated by WT SCADA data [16] is not sensitive enough to the defect when the defect is in its infancy. Owing to this reason, the available blade $\mathrm{CM}$ techniques that rely on interpreting the root bending moment signals have limitation in detecting incipient defects in addition to their inefficiency in damage location;

- Because of the high damping of the composite materials used in blades, the acoustic emission and ultrasonic waves are significantly damped in the process of wave transmission. Consequently, the acoustic emission and ultrasonic sensors cannot receive effective CM signals if they are mounted far from the position of the defect. In addition, both acoustic emission and ultrasonic CM signals need to be collected by using a high sampling frequency (usually $\geq 20 \mathrm{kHz}$ ). This means that a large 
amount of data will be collected by the data acquisition system within a short time, and consequently a big data 'mountain' will be formed in the end of the 20 to 30 years service life of a blade. Then, how to manage and analyze these data becomes a big challenge to the operator;

- WT blades are subjected to constantly varying loads in operation. The varying loads and structural damage are always coupled together to take effect on the dynamic response of the blade [9]. As it is unlikely to decouple their effects, the varying loads can modulate the CM signals and consequently lead to false alarms, which not only discount the added value of the $\mathrm{CM}$ systems but increase the unnecessary site visit, prolong the downtime, and enlarge the revenue loss [17]. So, how to develop a load-independent blade CM technique is one of the tough tasks in wind power industry today;

- The energy capturing performance/efficiency is often used as an indicator of the structural health condition of WT blades [9][18]. However, the degeneration in performance/efficiency does not necessarily mean the presence of structural damage in a blade. For instance, the ice and snow built up on blade surfaces can change the surface roughness, thus decrease the energy capturing efficiency of the blade. In such a circumstance, the degenerated performance/efficiency can recover automatically once the ice and snow on the blade melt at warm air temperatures. But the degeneration caused by structural damage or the poor surface roughness due to erosion and corrosion will be permanent and unrecoverable. Up to date, how to distinguish the true reason of the performance/efficiency degeneration of a WT blade and determine whether it is necessary to take action to deal with the change is a challenging issue to solve.

The aforementioned limitations do challenge the reliability of blade $\mathrm{CM}$ results. However, in view of the potentially significant contribution of reliable $\mathrm{CM}$ in improving the economic return of WTs, it is of significance to make effort to achieve such a technique particularly before the massive deployment of WTs in the following years. The work presented in this paper is part of the effort for reaching such an objective.

The paper proposes a new blade CM method based on the concept of the transmissibility of Frequency Response Functions (FRFs) [19][20]. The novelties can be briefly summarised as follows: (1) the ratios of the frequency responses are used for the calculation of CM criterion, which, to certain extent, can mitigate the effect of varying loads and therefore improves the reliability of blade CM result; (2) damage location can be easily achieved via the proposed technique thanks to the advantage of the FRF transmissibility analysis; consequently, the value of the CM criterion is not only a reliable indicator of the actual health condition of the blade but a useful tool for accurate damage location; (3) since the proposed CM criterion responds only to the degenerated performance/efficiency caused by structural damage, the false alarms due to the ice and snow built up on blade surfaces can be fully avoided; and (4) the proposed technique is able to work very well despite the types of the sensors that are used for data acquisition. In this study, the effectiveness of the proposed technique in blade $\mathrm{CM}$ and damage location has been verified by applying the technique to monitoring the structural integrity of a full scale WT blade during its fatigue and static tests. More details about this work are depicted in Sections II and III.

Potentially, the CM technique developed in this paper is applicable to two scenarios in wind power practice:

(1) Laboratory test of WT blades. Nowadays, a number of WT blade testing centres, such as NREL, Narec, VMC, SGS, and Denmark's National Test Centre, are operating commercially to accelerate the design and development of long blades for giant WTs with the capacity of $>5 \mathrm{MW}$. In blade tests, visual inspection is often used for structural health assessment, which consumes time and moreover is difficult to find incipient defects occurring in the blades [21]. The technique proposed in this paper can help to accomplish the blade CM and fault detection efficiently via interpreting the measured signals online;

(2) CM WT blades in operation. The technique proposed in this paper is superior to the existing WT blade CM techniques in both fault detection and fault location as long as the required CM information along blade span can be collected properly. For those WT blades in operation, the kind of information along blade span can be collected by various means, such as distributed strain/stress transducers [22]. However, it is aware that the installation of distributed strain/stress transducers on operational blades will be a tough issue. To allow an easy data acquisition, a non-contact measurement method with the aid of stereo imaging camera is being studied by the authors. The relevant research achievements will be reported separately.

\section{METHODOLOGY}

WT blades are typically constructed using fibrereinforced polymeric composites and sandwich structures. Moreover, their geometries (e.g. the aerofoil chord length) vary gradually along span-wise direction. It is therefore a challenging task to develop an accurate analytical model for WT blades. For simplification, in the present study, we regard a WT blade to be a multi-degree-of-freedom (MDOF) system consisting of a number of sections, as shown in Fig.1.

In Fig.1, three neighbouring sections are denoted as sections $i-1$, $i$ and $i+1$ with masses $m_{i-1}, m_{i}$ and $m_{i+1}$, respectively. The sections $\mathrm{i}-1$ and $\mathrm{i}$ are connected via stiffness $\mathrm{k}_{\mathrm{i}-1, \mathrm{i}}$ and damping $\mathrm{c}_{\mathrm{i}-1, \mathrm{i}}$; and the sections $\mathrm{i}$ and $\mathrm{i}+$ 1 are connected via $k_{i, i+1}$ and $c_{i, i+1}$. Consequently, when an external load, either distributed or concentrated, is applied 
to the blade, the dynamic response of the blade can be expressed by the following equation:

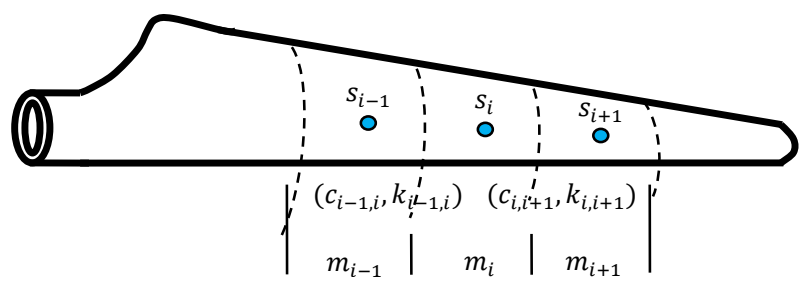

Fig.1 The model of a wind turbine blade.

$$
\mathbf{M} \ddot{\mathbf{X}}(t)+\mathbf{C} \dot{\mathbf{X}}(t)+\mathbf{K X}(t)=\mathbf{F}(t)
$$

where, $\mathbf{X}(\mathbf{t})$ represents the vector of displacement responses along the blade, $\mathbf{M}, \mathbf{C}$ and $\mathbf{K}$ denote the mass, damping and stiffness matrices, respectively, $\mathbf{F}$ is the matrix of external forces.

It can be inferred that when a local defect occurs in section $\mathrm{i}$, the values of $\mathrm{c}_{\mathrm{i}-1, \mathrm{i}}, \mathrm{c}_{\mathrm{i}, \mathrm{i}+1}, \mathrm{k}_{\mathrm{i}-1, \mathrm{i}}$ and $\mathrm{k}_{\mathrm{i}, \mathrm{i}+1}$ will change correspondingly; whilst the damping and stiffness in other blade sections may not change. This inspires the thought of this paper to develop a damage detection and location technique for WT blades by the approach of FRF transmissibility analysis. Since the corresponding values of damping and stiffness are dependent only on the structural integrity of the blade, the CM technique developed based on this idea will respond only to those changes caused by structural damage. Thanks to this merit of the technique, the false alarms due to the ice and snow built up on blade surfaces can be fully avoided.

Assume when an external force $f(t)$ is applied to the blade, $x_{i}(t)$ and $x_{k}(t)\left(t=t_{0}, t_{1}, \ldots, t_{N-1}\right)$ are the data series measured by two neighboring sensors mounted on the blade. The frequency spectra of $f(t), x_{i}(t)$ and $x_{k}(t)$ can be readily obtained by performing the following Discrete Fourier transforms (DFT):

$$
\left\{\begin{array}{l}
\mathcal{F}_{f}(j \omega)=\sum_{n=0}^{N-1} f\left(t_{n}\right) e^{-j n \omega / f_{s}} \\
\mathcal{F}_{x_{i}}(j \omega)=\sum_{n=0}^{N-1} x_{i}\left(t_{n}\right) e^{-j n \omega / f_{s}} \\
\mathcal{F}_{x_{k}}(j \omega)=\sum_{n=0}^{N-1} x_{k}\left(t_{n}\right) e^{-j n \omega / f_{s}}
\end{array}\right.
$$

where $f_{s}$ refers to sampling frequency, and $N$ is the total number of data used for the DFT analysis.

Denote the FRFs of the $i$-th and $k$-th blade sections with respect to the external force $f(t)$ as $R_{x_{i}}(j \omega)$ and $R_{x_{k}}(j \omega)$, then the frequency component of $\mathcal{F}_{x_{i}}(j \omega)$ and $\mathcal{F}_{x_{k}}(j \omega)$ at the $r$-th frequency $\omega_{r}$ can be written as

$$
\left\{\begin{array}{l}
\mathcal{F}_{x_{i}}\left(j \omega_{r}\right)=R_{x_{i}}\left(j \omega_{r}\right) \mathcal{F}_{f}\left(j \omega_{r}\right) \\
\mathcal{F}_{x_{k}}\left(j \omega_{r}\right)=R_{x_{k}}\left(j \omega_{r}\right) \mathcal{F}_{f}\left(j \omega_{r}\right)
\end{array}\right.
$$

then in accordance with the concept of the transmissibility of the FRFs [19], the transmissibility of the FRFs at frequency $\omega_{r}$ can be described as

$$
T_{i, k}\left(j \omega_{r}\right)=\frac{R_{x_{i}}\left(j \omega_{r}\right)}{R_{x_{k}}\left(j \omega_{r}\right)}
$$

$$
\begin{aligned}
& =\frac{\mathcal{F}_{x_{i}}\left(j \omega_{r}\right) / \mathcal{F}_{f}\left(j \omega_{r}\right)}{\mathcal{F}_{x_{k}}\left(j \omega_{r}\right) / \mathcal{F}_{f}\left(j \omega_{r}\right)} \\
& =\frac{\mathcal{F}_{x_{i}}\left(j \omega_{r}\right)}{\mathcal{F}_{x_{k}}\left(j \omega_{r}\right)}
\end{aligned}
$$

This indicates that $T_{i, k}\left(j \omega_{r}\right)$ can be estimated directly from the spectra $\mathcal{F}_{x_{i}}\left(j \omega_{r}\right)$ and $\mathcal{F}_{x_{k}}\left(j \omega_{r}\right)$. In order to minimize the negative effects of measurement errors, multiple tests are often carried out for reaching an average transmissibility, i.e.

$$
\begin{aligned}
T_{i, k}\left(j \omega_{r}\right) & =\frac{1}{M} \sum_{m=1}^{M} T_{i, k}^{m}\left(j \omega_{r}\right) \\
& =\frac{1}{M} \sum_{m=1}^{M}\left(\frac{\mathcal{F}_{x_{i}}^{m}\left(j \omega_{r}\right)}{\mathcal{F}_{x_{k}}^{m}\left(j \omega_{r}\right)}\right)
\end{aligned}
$$

where, the superscript $m=1,2, \ldots, M$ indicates the number of tests.

Use the transmissibility of the FRFs obtained when the blade has perfect structural integrity as benchmark, then a new CM criterion $C_{i, k}$ can be defined as

$$
C_{i, k}=\frac{1}{N} \sum_{r=1}^{N}\left[T_{i, k}\left(j \omega_{r}\right)-T_{i, k}^{*}\left(j \omega_{r}\right)\right]
$$

where $T_{i, k}^{*}\left(j \omega_{r}\right)$ refers to the transmissibility of the FRFs at frequency $\omega_{r}$ obtained when the blade is perfect in integrity.

From the above equations, it can be inferred that:

- Instead of investigating the variation tendencies of individual CM signal in either frequency or amplitude, the transmissibility analysis realizes blade CM by monitoring the correlation of different blade sections in dynamic response. Since the correlation between the measured responses of different sections relies on the local material and structural properties of the blade, the $C_{i, k}$ will respond as long as the damage results in changes in these local properties of the blade;

- An evident change in the value of $\mathrm{CM}$ criterion $C_{i, k}$ will indicate the presence of structural defect in the vicinity of either sensor $s_{i}$ or $s_{k}$. The further development of the defect will be characterized by the increasing tendency of the value of $C_{i, k}$;

- As the transmissibility of the FRFs reflects the relationship between the frequency responses of neighbouring blade sections, the simultaneous increase of the values of $C_{a, b}$ and $C_{b, c}$ suggests that a defect is present in the blade section where the sensor ' $b$ ' is installed. This implies that the proposed CM criterion has an attractive damage location capability. Moreover, the comparison of the values of $C_{a, b}$ and $C_{b, c}$ can further enhance such a capability and lead to a more accurate prediction to the position of the defect. As Fig.2 shows, $C_{a, b}>C_{b, c}$ indicates that the defect locates at the left side (i.e. the side of sensor ' $a$ '), while $C_{b, c}>C_{a, b}$ indicates that the defect locates at the right side (i.e. the side of sensor ' $c$ '). 


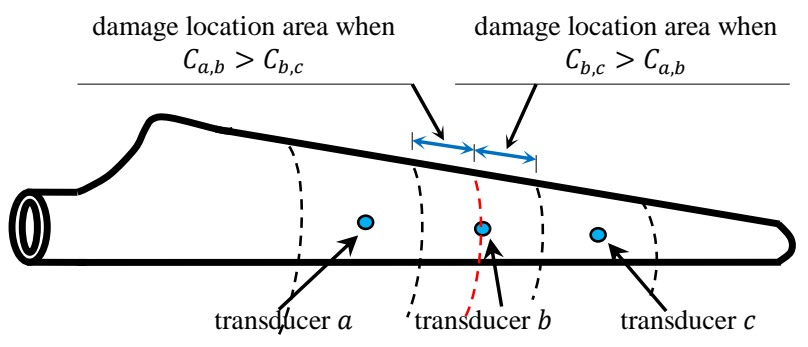

Fig.2 The prediction to the damage location.

\section{VERIFICATION EXPERIMENTS}

To verify the proposed technique, experiments paired with the fatigue and static tests of a full scale WT blade were conducted. The blade being tested was over $40 \mathrm{~m}$ long. Its natural frequency in flap direction was $0.83 \mathrm{~Hz}$. As Fig.3 shows, part of the tip section of the blade had been cut off in advance before the test, so that the blade would not touch the ground at large deflections.

$A C C O-A C C 1-A C C 2-A C C 3-A C C 4-A C C 5$

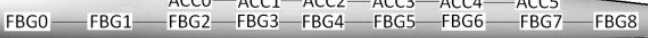

Fig.3 Transducers installed on the blade.

In the experiments, two sets of sensors were mounted on the blade. The first set consists of 9 Fibre Bragg Grating (FBG) sensors; and the second comprises 6 accelerometers. Both sets of sensors were installed along the span-wise direction of the blade. In view of defects are most likely to occur in the loading area of the blade, the sensors were deployed mainly in this area (see Fig.3). In addition, for facilitating data management two separate data acquisition systems were used in the test. One was for collecting data from FBG strain gauges by using a sampling frequency of $20 \mathrm{~Hz}$; another for collecting data from accelerometers by using a sampling frequency of $100 \mathrm{~Hz}$.

It is worth noting that the fatigue test of a full scale blade usually lasts for a few weeks. During the period, regular breaks are often arranged for visual inspection and system maintenance. During the period of the test, data were collected from the FBG strain gauges when the fatigue test was running; while the accelerometer data were collected only when the hammer-striking tests were conducted during the break of fatigue tests for checking blade health status.

As the purpose of the tests was to verify the long-term reliability of the blade being tested and assess its capability of withstanding ultimate loads, a static test of the blade was accordingly conducted after the blade experienced $10^{8}$ fatigue cycles in flap direction. Following the static test, more fatigue tests were performed again so that the extreme life cycle of the blade could be acquired. Fig.4 is an illustration of the static test and relevant facilities.

From Fig.4, it is seen that the section of the blade where the sensors FBG5 and ACC3 were mounted suffers large tension and compressive stresses in the static test, which directly resulted in the de-bonding of the adhesive joint between the spar beam and the aero-shell of the blade. Accordingly, defects were observed first in this area in the subsequent fatigue tests.

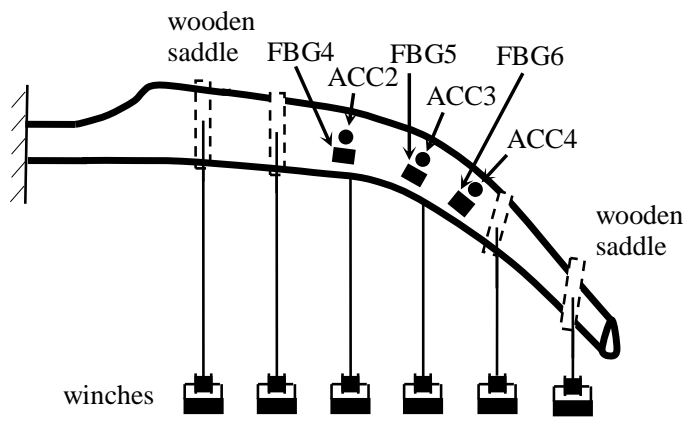

Fig. 4 Schematics of static test of the blade.

The observation shows that in the experiments, the incipient defect was characterized by a number of swells and ripples emerging in a local region of the surface of the blade. Then, these swells and ripples evolved to be cracks, which propagate along the fibres of the composite material, as shown in Fig.5. a)

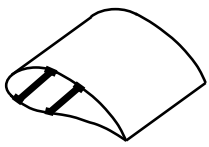

d)

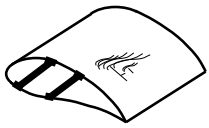

b)

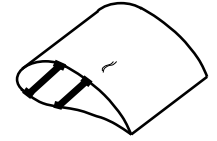

e)

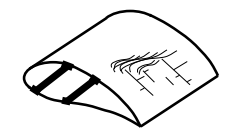

c)

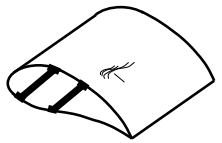

Fig.5 Demonstration of surface cracks. (a) Perfect blade; (b) Severity level 1; (c) Severity level 2; (d) Severity level 3; (e) Severity level 4.

As a consequence of the growth of these cracks, the structural integrity of the blade starts to degenerate gradually. However, when the degeneration is minor it can hardly be detected through observing the time waveforms or the frequencies of the CM signals. This is why available blade CM methods often show inefficiency in detecting infantile defects. For this reason, the research reported in this paper is an effort to detect the incipient blade defect thus protects the defective blade early and avoids catastrophic failure.

Considering a large amount of data collected from both sets of sensors FBG0-FBG8 and ACC0-ACC5 (see Fig.3) will be used to calculate the $\mathrm{CM}$ criterion $C_{i, k}$ in this verification experiment and it is unlikely to present all these data in the limited context of this paper, an illustrative example of these data are shown in Fig.6 for the purpose of demonstration. Where, Fig.6a shows the example data collected from the strain gauges FBG0-FBG8, and Fig.6b shows the data from the accelerometers ACC0-ACC5. The corresponding frequency spectra of these signals obtained by the DFT are shown in Fig.7 in order to disclose the frequency compositions of these CM signals. 
a)
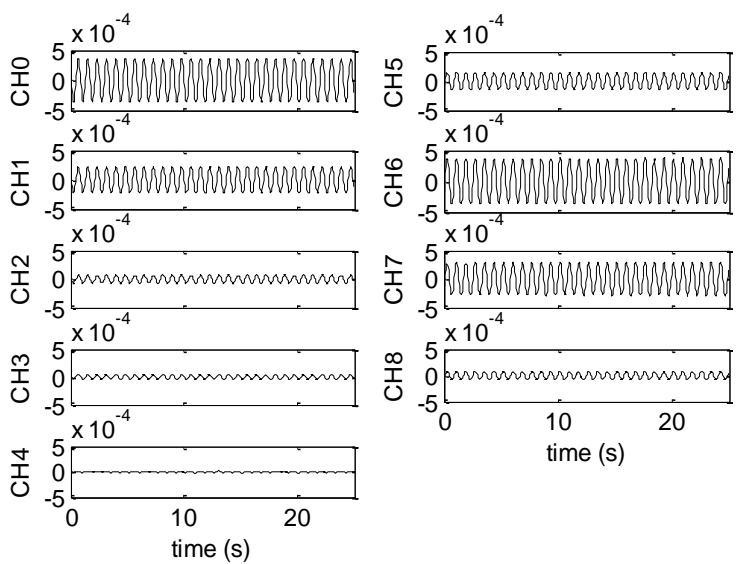

b)

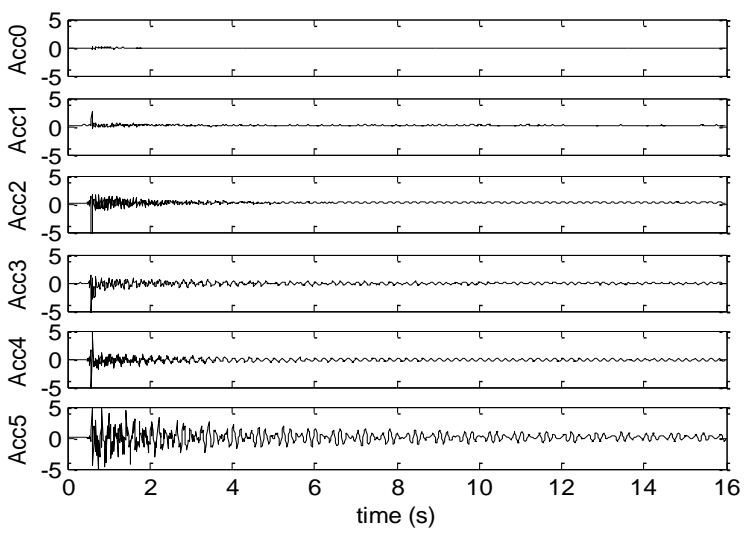

Fig.6 Sample data. (a) Data from the FBG strain gauges; (b) Data from accelerometers.

a)
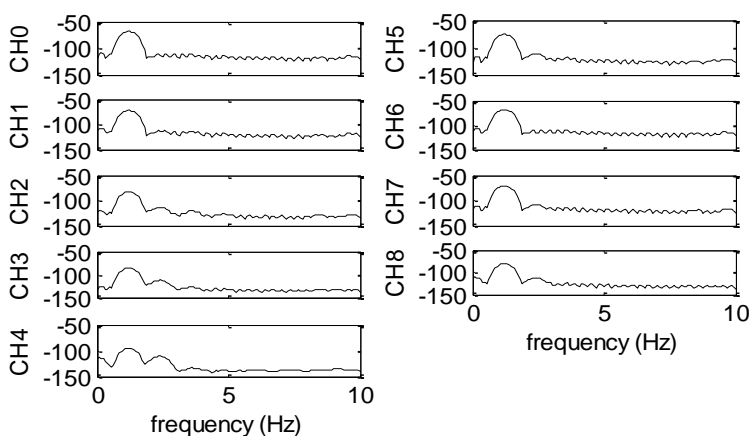

b)

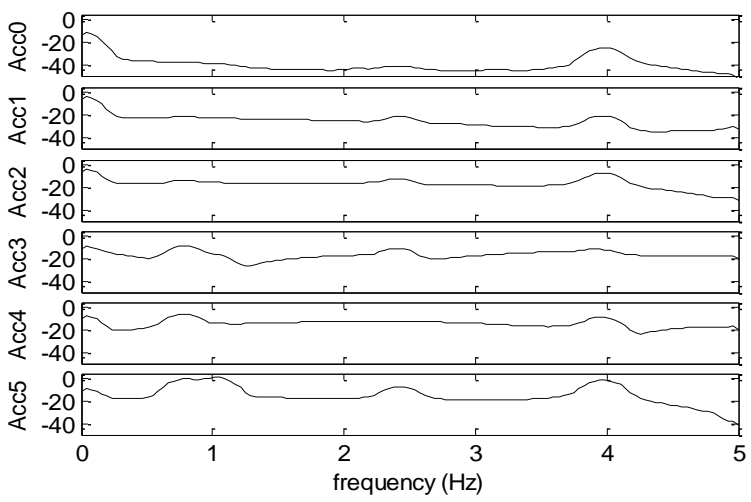

Fig.7 Frequency spectra of the sample data. (a) For data from the FBG strain gauges; (b) For data from accelerometers.
Herein, it is worth noting that in the hammer-striking tests, the region near the sensors FBG7 and ACC5 was selected as the hammer-striking position based on the assumption that the slim section near blade tip can produce better response to the strike than the thicker section at blade root does.

From the example data shown in Figs. 6 and 7, it is seen that the FBG strain gauge signals collected from different locations are different in amplitude. But their oscillations are dominated by the same frequency, which is pre-set in the control system for controlling the flapping period of the blade in the fatigue test. Since resonant testing [21] was adopted in the fatigue test. The frequency observed from Fig.7a should be the same or at least close to the natural frequency of the blade. The value of the frequency can be also perceived from the results shown in Fig.7b, although the acceleration signals collected from the far locations from the hammer-striking position (i.e. Acc0, Acc1, and Acc2) are unable to indicate this frequency very well.

To verify the CM capability of the proposed technique, 105 sets of FBG strain gauge data and 20 sets of acceleration data collected in different structural integrity circumstances were selected to fulfil the purpose of verification, see Table 1 . As defect was never observed before the static test, the data collected before the static test was used for calculating the benchmark transmissibility of the FRFs $T_{i, k}^{*}\left(j \omega_{r}\right)$ in (6), the data obtained after the static test were used for calculating the transmissibility function $T_{i, k}\left(j \omega_{r}\right)$.

Table 1. The data used for verification.

\begin{tabular}{c|c|c}
\hline $\begin{array}{c}\text { Health condition } \\
\text { of the blade }\end{array}$ & $\begin{array}{c}\text { Number of data sets } \\
\text { collected from FBG } \\
\text { strain gauges }\end{array}$ & $\begin{array}{c}\text { Number of data sets } \\
\text { collected from } \\
\text { accelerometers }\end{array}$ \\
\hline $\begin{array}{c}\text { Health blade } \\
\text { Blade with the } \\
\text { defect of level 1 } \\
\text { Blade with the } \\
\text { defect of level 2 } \\
\text { Blade with the } \\
\text { defect of level 3 }\end{array}$ & 20 & 4 \\
$\begin{array}{c}\text { Blade with the } \\
\text { defect of level 4 }\end{array}$ & 20 & 4 \\
\hline Total & 10 & 4 \\
\hline
\end{tabular}

As Table 1 shows, the selected data cover the entire development process of the defects (i.e. a cluster of cracks emerging on the surface of the blade) from their initial to further propagation. The cracks were located near the sensors FBG5 and ACC3 and at the side of sensors FBG4/ACC2 (see Fig.8).

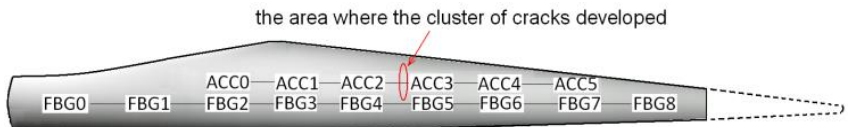

Fig.8 The location of the defects. 
Assume the blade is perfect in structural integrity before the static test and use the data measured at the early fatigue and hammer-striking tests as benchmark data. Set the number of tests defined in (5) to be $M=2$, then the 105 FBG strain gauge data and 20 accelerometer data listed in Table 1 were calculated by using the methodology described in Section II. The calculation results are shown in Fig.9.

a)

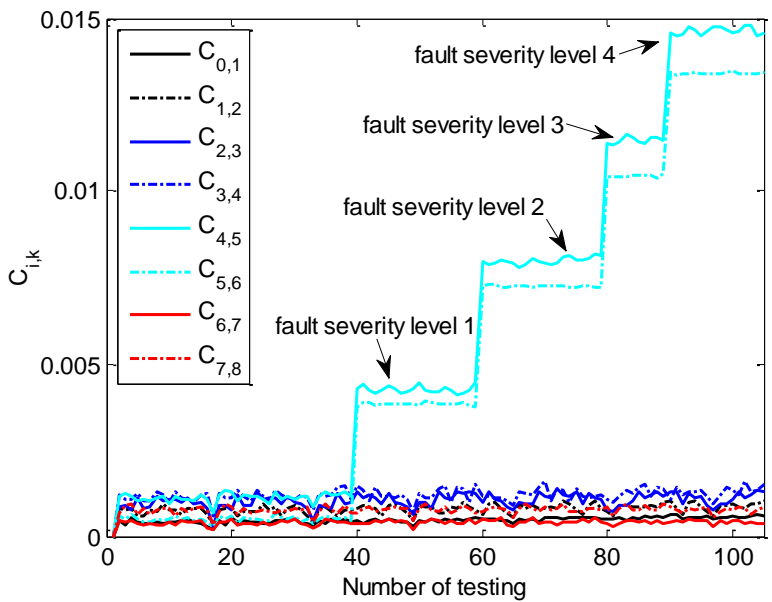

b)

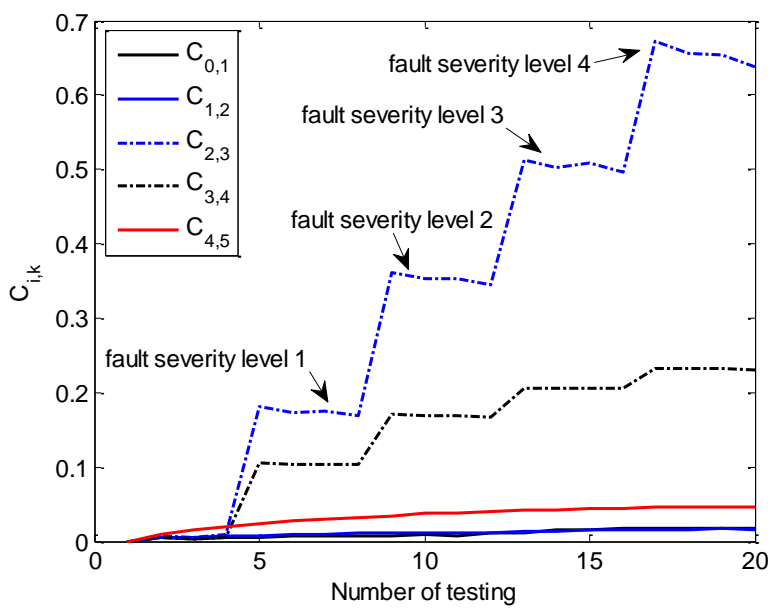

Fig.9 Blade condition monitoring results. (a) from the FBG strain gauge data; (b) from the acceleration data.

From Fig.9, it is found that:

- The proposed technique works very well whatever the FBG strain gauges or the accelerometers are used as the sensors for data acquisition. In the absence of the defects, the CM criterion $C_{i, k}$ does not show evident change in value;

- The CM criterion $C_{i, k}$ is alert to the presence of the defects. Its value shows a significant increase immediately as soon as the defects are present. Moreover, the value increases gradually with the continual propagation of the cracks;

- The resultant $C_{i, k}$ shown in Fig.9a suggests that the defects are located near the sensor FBG5; and the resultant $C_{i, k}$ shown in Fig.9b suggests that the defects are near accelerometer ACC3. By referring to Fig.8, it is found that both sets of CM data give a correct prediction to the position of the defects;

- In the presence of the defects, $\mathrm{C}_{4,5}>\mathrm{C}_{5,6}$ in Fig.9a and $\mathrm{C}_{2,3}>\mathrm{C}_{3,4}$ in Fig.9b. This indicates that the defects are located near the sensors FBG5/ACC3 and at the side of the sensors FBG4/ACC2. Obviously, such a prediction very well matches the observation shown in Fig.8.

\section{IV.CONCLUDING REMARKS}

Large modern WTs require reliable CM techniques to assure their long-term reliability, availability and economic return. The research depicted in this paper is part of the effort for meeting such a need. With the aid of the concept of the transmissibility of FRFs, a new CM technique has been developed dedicatedly for monitoring long WT blades. Experimental data analysis has shown that the proposed technique is effective not only in detecting the defects occurring in a blade but in locating their positions when either FBG strain gauges or accelerometers are used as the sensors for data acquisition. However, since the proposed $\mathrm{CM}$ criterion responds only to the blade performance/efficiency degeneration caused by structural damage, the proposed technique cannot be applied to detect the ice and snow built up on blade surfaces. In addition, as a long WT blade is essentially a complicated nonlinear MDOF system, the system nonlinearities would significantly amplify the effect of external loads on the CM results. Therefore, how to apply the lab research achievements to the practical CM of long WT blades in operation is still an issue worthy to further study. To address these issues, further researches are undergoing. The relevant achievements will be reported in future publications.

\section{REFERENCES}

[1] J. Ribrant and L. Bertling, "Survey of failures in wind power systems with focus on Swedish wind power plants during 1997-2005," IEEE Transactions on Energy Conversion, vol.22, no.1, pp 167-173, 2007.

[2] P. Tavner, Offshore Wind Turbine: Reliability, Availability \& Maintenance. IET Press, ISBN: 978-1-84919-229-3, 2012.

[3] K. Fischer, F. Besnard, and L. Bertling, "Reliability-centered maintenance for wind turbines based on statistical analysis and practical experience," IEEE Transactions on Energy Conversion, vol. 27, no. 1, pp 184-195, 2012.

[4] F. Besnard and L. Bertling, "An approach for condition-based maintenance optimization applied to wind turbine blades," IEEE Transactions on Sustainable Energy, vol.1, no.2, pp 77-83, 2010.

[5] P. Caselitz and J. Giebhardt, "Rotor condition monitoring for improved operational safety of offshore wind energy converters," ASME Journal of Solar Energy Engineering, vol.127, pp 253-261, 2005.

[6] C.C. Ciang, J.R. Lee, and H.J. Bang, "Structural health monitoring for a wind turbine system: a review of damage detection methods," Measurement Science and Technology, vol.19, pp 1-20, 2008.

[7] Y. Amirat, M.E.H. Benbouzid, E. Al-Ahmar, B. Bensaker, and S. Turri, "A brief status on condition monitoring and fault diagnosis in wind energy conversion systems," Renewable and Sustainable Energy Reviews, vol.13, pp 2629-2636, 2009.

[8] W. Yang, P. Tavner, C. Crabtree, Y. Feng, and Y. Qiu, "Wind turbine condition monitoring: technical and commercial challenges," Wind Energy, vol.17, no.5, pp 673-693, 2014. 
[9] W. Yang, R. Court, and J. Jiang, "Wind turbine condition monitoring by the approach of SCADA data analysis," Renewable Energy, vol.53, pp 365-376, 2013.

[10] C.S. Tsai, C.T. Hsieh, and S.J. Huang, "Enhancement of damagedetection of wind turbine blades via CWT-based approaches," IEEE Transactions on Energy Conversion, vol.21, no.3, pp 776-781, 2006.

[11] W.Q. Jeffries, J.A. Chambers, and D.G. Infield, "Experience with bicoherence of electrical power for condition monitoring of wind turbine blades," IEE Proceedings Vision, Image and Signal Processing, vol. 145, no.3, pp 141-148, 1998.

[12] C.S. Shin, B.L. Chen, and S.K. Liaw, "Foreign object impact monitoring on wind turbine blade using FBGs," in Proceedings of IEEE Conference of Sensors, Limerick, October 2011.

[13] B. Lu, Y. Li, X. Wu, and Z. Yang, "A review of recent advances in wind turbine condition monitoring and fault diagnosis," in Proceedings of IEEE Power Electronics and Machines in Wind Applications (PEMWA), Lincoln, June 2009.

[14] G. Aranguren, P.M. Monje, V. Cokonaj, E. Barrera, and M. Ruiz, "Ultrasonic wave-based structural health monitoring embedded instrument," Review of Scientific Instruments, vol.84, no. 12, pp 125106-125106-7, 2013.

[15] X. Gong and W. Qiao, "Imbalance fault detection of direct-drive wind turbines using generator current signals," IEEE Transactions on Energy Conversion, vol.27, no.2, pp 468-476, 2012.

[16] Joon-Young Park, Jae-Kyung Lee, Ki-Yong Oh, Jun-Shin Lee, "Development of a novel power curve monitoring method for wind turbines and its field tests," IEEE Transactions on Energy Conversion, vol.29, no.1, pp 119-128, 2014.

[17] Y. Qiu, Y. Feng, P. Tavner, P. Richardson, G. Erdos, and B. Chen, "Wind turbine SCADA alarm analysis for improving reliability," Wind Energy, vol.15, no.8, pp 951-966, 2012.

[18] S. Gill, B. Stephen, and S. Galloway, "Wind turbine condition assessment through power curve copula modeling," IEEE Transactions on Sustainable Energy, vol.3, no. 1, pp 94-101, 2012.

[19] Z. Lang, G. Park, C. Farrar, M. Todd, Z. Mao, L. Zhao, and K. Worden, "Transmissibility of non-linear output frequency response functions with application in detection and location of damage in MDOF structural systems," International Journal of Non-linear Mechanics, vol.46, pp 841-853, 2011.

[20] Z. Lang and S.A. Billings, "Energy transfer properties of nonlinear systems in the frequency domain," International Journal of Control, vol.78, pp 354-362, 2005.

[21] W. Yang, "Testing and condition monitoring of composite wind turbine blades," in Recent Advances in Composite Materials for Wind Turbines Blades, Dr. Brahim Attaf (Ed.), ISBN 978-0-9889190-0-6, The World Academic Publishing Co. Ltd, December 2013.

[22] M. Moradi, S. Sivoththaman, "MEMS multisensor intelligent damage detection for wind turbines," IEEE Sensors Journal, vol.15, no.3, pp 1437-1444, 2014. 